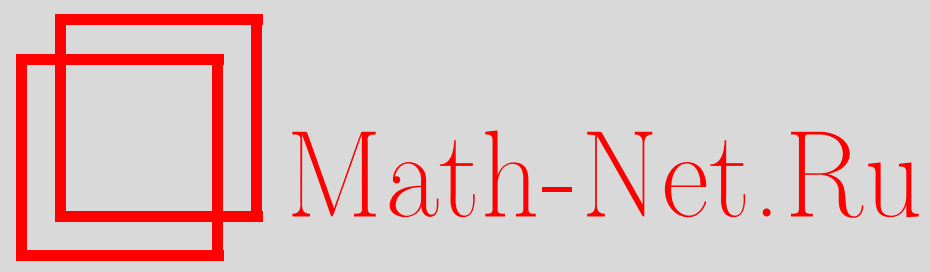

В. А. Емеличев, К. Г. Кузьмин, Оценки радиуса устойчивости векторной задачи о максимальном разрезе графа, Дискрет. матем., 2013, том 25, выпуск 2, 5-12

DOI: https://doi.org/10.4213/dm1230

Использование Общероссийского математического портала Math-Net.Ru подразумевает, что вы прочитали и согласны с пользовательским соглашением http://www.mathnet.ru/rus/agreement

Параметры загрузки:

IP: 52.23 .180 .231

26 апреля 2023 г., 12:56:39 


\title{
Оценки радиуса устойчивости векторной задачи \\ о максимальном разрезе графа
}

() 2013 г. В. А. Емеличев, К. Г. Кузьмин

\begin{abstract}
Получены нижняя и верхняя достижимые оценки радиуса устойчивости векторного варианта задачи о максимальном разрезе графа в случае, когда в пространстве параметров задачи задана метрика Гельдера.

Работа выполнена при поддержке Белорусского республиканского фонда фундаментальных исследований, проект Ф11K-095.
\end{abstract}

Один из известных подходов к исследованию проблем устойчивости векторных задач дискретной оптимизации ориентирован на получение количественных оценок, характеризующих меру устойчивости. В основу этого подхода положено ключевое понятие радиуса устойчивости задачи, под которым понимается предельный уровень возмущений параметров задачи в метрическом пространстве, не приводящих к появлению новых Парето-оптимальных решений. Большинство результатов, полученных в этом направлении, связано с нахождением формул или оценок радиуса устойчивости векторных задач булева или целочисленного программирования с линейными $[1,2,3,4,5,6]$ и минимаксными (максиминными) $[7,8,9,10]$ критериями.

В данной работе проведен анализ устойчивости в метрике Гельдера многокритериального варианта известной (см., например, $[11,12])$ комбинаторной экстремальной задачи о максимальном разрезе графа (MAX-CUT problem), являющейся классической проблемой дискретной оптимизации с многочисленными приложениями $[13,14,15]$. Получены верхняя и нижняя достижимые оценки радиуса устойчивости рассматриваемой задачи, сформулированной как задача булева квадратичного программирования.

Рассмотрим связный помеченный простой $(n, m)$-граф $G=(V, E)[16]$ с множеством вершин $V=N_{n}=\{1,2, \ldots, n\}$ и множеством ребер $E$, занумерованных числами $r \in N_{m}$. Пусть $(S, \bar{S})$ - разбиение множества $V$ вершин графа $G$ на два не пересекающихся непустых подмножества $S$ и $\bar{S}$. Тогда множество ребер $\{i, j\}$ графа $G$, концевые вершины которых находятся в разных подмножествах, называется разрезом графа и обозначается $(S, \bar{S})$. Всякий разрез $(S, \bar{S})$ графа $G$ порождает булев вектор $x=\left(x_{1}, x_{2}, \ldots, x_{n}\right) \in \mathbf{E}^{n}=\{0,1\}^{n}$ с компонентами

$$
x_{i}=\left\{\begin{array}{l}
1, \quad \text { если } i \in S, \\
0, \quad \text { если } i \in \bar{S} .
\end{array}\right.
$$

Ясно, что всякому вектору $x \in X=\mathbf{E}^{n} \backslash\left\{\mathbf{0}^{(n)}, \mathbf{1}^{(n)}\right\}$, где $\mathbf{0}^{(n)}=(0,0, \ldots, 0) \in \mathbf{E}^{n}$, $\mathbf{1}^{(n)}=(1,1, \ldots, 1) \in \mathbf{E}^{n}$, соответствует разрез $(S, \bar{S})$ с подмножествами $S=\{i \in V$ : 
$\left.x_{i}=1\right\}$ и $\bar{S}=\left\{i \in V: x_{i}=0\right\}$. В частности, вектору $\bar{x}=\mathbf{1}^{(n)}-x \in X$ соответствует разрез $(\bar{S}, S)$. Всякий вектор $x \in X$ естественно называть разрезом графа.

Далее все ребра $\{i, j\}$ графа $G$ будем ориентировать так, что $E \subseteq\{(i, j): 1 \leqslant i<$ $j \leqslant n\}$, т. е. превратим множество ребер в множество дуг. Каждой дуге $(i, j) \in E$ с номером $r \in N_{m}$ сопоставим вектор-столбец $\left(w_{i j}^{1}, w_{i j}^{2}, \ldots, w_{i j}^{s}\right)^{T}$, где $w_{i j}^{k} \in \mathbf{R}-$ вес дуги $(i, j)$, соответствующий критерию $k \in N_{s}$. Из этих $m$ столбцов, упорядоченных в соответствии с порядком дуг множества $E$, образуем матрицу $W=\left[w_{i j}^{k}\right] \in \mathbf{R}^{s \times m}$ со строками $W_{k} \in \mathbf{R}^{m}, k \in N_{s}$. Учитывая нумерацию дуг графа $G$, матрицу $W$ удобно иногда представлять в виде $W=\left[w_{k r}\right] \in \mathbf{R}^{s \times m}$. Тогда $W_{k}=\left(w_{k 1}, w_{k 2}, \ldots, w_{k m}\right)$, $k \in N_{s}$. В этих обозначениях легко видеть, что квадратичная функция

$$
f_{k}\left(x, W_{k}\right)=\sum_{(i, j) \in E} w_{i j}^{k}\left(x_{i}-x_{j}\right)^{2},
$$

заданная на множестве векторов $X$, представляет собой суммарный вес разреза $(S, \bar{S})$ по критерию $k$, где $S=\left\{i \in V: x_{i}=1\right\}, \bar{S}=\left\{i \in V: x_{i}=0\right\}$. В результате возникает векторный ( $s$-критериальный) вариант классической задачи о максимальном разрезе графа

$$
Z^{s}(W): \quad f(x, W)=\left(f_{1}\left(x, W_{1}\right), f_{2}\left(x, W_{2}\right), \ldots, f_{s}\left(x, W_{s}\right)\right) \rightarrow \max _{x \in X}
$$

состоящей в поиске множества Парето, т. е. множества эффективных разрезов

$$
P^{s}(W)=\{x \in X: X(x, W)=\varnothing\},
$$

где

$$
X(x, W)=\left\{x^{\prime} \in X: f(x, W) \leqslant f\left(x^{\prime}, W\right) \& f(x, W) \neq f\left(x^{\prime}, W\right)\right\} .
$$

Легко видеть, что $x \in P^{s}(W)$ тогда и только тогда, когда $\bar{x}=\mathbf{1}^{(n)}-x \in P^{s}(W)$, поэтому $\left|P^{s}(W)\right|$ - четное число.

Для всякого натурального числа $d$ в действительном пространстве $\mathbf{R}^{d}$ зададим метрику Гельдера $l_{p}, p \in[1, \infty]$, т. е. под нормой вектора $y=\left(y_{1}, y_{2}, \ldots, y_{d}\right) \in \mathbf{R}^{d}$ будем понимать число

$$
\|y\|_{p}= \begin{cases}\left(\sum_{i \in N_{d}}\left|y_{i}\right|^{p}\right)^{1 / p}, & \text { если } 1 \leqslant p<\infty, \\ \max _{i \in N_{d}}\left|y_{i}\right|, & \text { если } p=\infty,\end{cases}
$$

а под нормой матрицы - норму вектора, составленного из всех ее элементов. Как известно, для любых векторов $u, v \in \mathbf{R}^{m}$ выполняется неравенство Гельдера

$$
|\langle u, v\rangle| \leqslant\|u\|_{p} \cdot\|v\|_{q},
$$

где $\langle u, v\rangle$ - скалярное произведение векторов $u$ и $v$, а величины $p$ и $q$ связаны соотношением $1 / p+1 / q=1$, при этом $q=1$, если $p=\infty$, и $q=\infty$, если $p=1$. Кроме того, полагаем $1 / p=0$ при $p=\infty$.

Используя известное условие [17], при котором неравенство (1) выполняется как равенство, нетрудно убедиться в справедливости следующей формулы

$$
\forall v \in \mathbf{R}^{m} \forall \theta>0 \exists u^{0} \in \mathbf{R}^{m} \quad\left(\left\|u^{0}\right\|_{p}=\theta \quad \& \quad\left|\left\langle u^{0}, v\right\rangle\right|=\theta\|v\|_{q}\right) .
$$


По аналогии с $[6,2,3,5,1,7,8,9,10]$ радиусом устойчивости задачи $Z^{s}(W)$, $s \in \mathbf{N}$, в метрике Гельдера $l_{p}, p \in[1, \infty]$, назовем число

$$
\rho(s, p)= \begin{cases}\sup \Theta_{p}, & \text { если } \Theta_{p} \neq \varnothing, \\ 0, & \text { если } \Theta_{p}=\varnothing,\end{cases}
$$

где

$$
\begin{gathered}
\Theta_{p}=\left\{\varepsilon>0: \forall U \in \Omega_{p}(\varepsilon) \quad\left(P^{s}(W+U) \subseteq P^{s}(W)\right)\right\}, \\
\Omega_{p}(\varepsilon)=\left\{U \in \mathbf{R}^{s \times m}:\|U\|_{p}<\varepsilon\right\},
\end{gathered}
$$

$\|U\|_{p}$ - норма Гельдера $l_{p}$ матрицы $U$. Множество $\Omega_{p}(\varepsilon)$ принято называть множеством возмущающих матриц.

Таким образом, радиус устойчивости задачи $Z^{s}(W)$ - это предельный уровень возмущений элементов матрицы $W$ в метрическом пространстве $\mathbf{R}^{s \times m}$, которые не приводят к появлению новых эффективных разрезов.

Легко видеть, что при $P^{s}(W)=X$ для всякой матрицы $W \in \mathbf{R}^{s \times m}$ включение $P^{s}(W+U) \subseteq P^{s}(W)$ имеет место при любой возмущающей матрице $U \in \mathbf{R}^{s \times m}$, т. е. $\rho(s, p)=\infty$. Задачу $Z^{s}(W)$, для которой $P^{s}(W) \neq X$, будем называть нетривиальной.

Каждому разрезу $x \in X$ и всякой дуге $(i, j) \in E$ с номером $r \in N_{m}$ поставим в соответствие число $\gamma_{r}(x)=\left|x_{i}-x_{j}\right|$. Кроме того, положим

$$
\begin{gathered}
\sigma_{r}\left(x^{\prime}, x\right)=\gamma_{r}\left(x^{\prime}\right)-\gamma_{r}(x), \quad r \in N_{m}, \\
\sigma\left(x^{\prime}, x\right)=\left(\sigma_{1}\left(x^{\prime}, x\right), \sigma_{2}\left(x^{\prime}, x\right), \ldots, \sigma_{m}\left(x^{\prime}, x\right)\right) .
\end{gathered}
$$

Тогда для любых разрезов $x, x^{\prime} \in X$ очевидны равенства

$$
\left\langle W_{k}, \sigma\left(x^{\prime}, x\right)\right\rangle=f_{k}\left(x^{\prime}, W_{k}\right)-f_{k}\left(x, W_{k}\right), \quad k \in N_{s} .
$$

Для $s \in \mathbf{N}$ и $p \in[1, \infty]$ введем обозначения

$$
\begin{gathered}
\varphi(s, p)=\min _{x \in X \backslash P^{s}(W)} \max _{x^{\prime} \in P(x, W)} \min _{k \in N_{s}} \frac{\left\langle W_{k}, \sigma\left(x^{\prime}, x\right)\right\rangle}{\left\|\sigma\left(x^{\prime}, x\right)\right\|_{q}}, \\
\psi(s, p)=\min \left\{\chi(s, p),(m s)^{1 / p} \varphi(s, \infty)\right\}, \\
P(x, W)=X(x, W) \cap P^{s}(W), \\
\chi(s, p)=\min _{k \in N_{s}}\left\|W_{k}\right\|_{p} .
\end{gathered}
$$

Легко видеть, что $\varphi(s, p) \geqslant 0$.

Теорема 1. При любых $s \in \mathbf{N} u p \in[1, \infty]$ для радиуса устойчивости нетривиальной задачи $Z^{s}(W)$ в метрике Гельдера $l_{p}$ справедливы оценки

$$
\varphi(s, p) \leqslant \rho(s, p) \leqslant \psi(s, p) .
$$

Доказательство. Вначале покажем справедливость неравенства $\rho(s, p) \geqslant \varphi(s, p)$. При $\varphi(s, p)=0$ оно очевидно. Пусть $\varphi(s, p)>0$ и пусть возмущающая матрица $U \in \mathbf{R}^{s \times m}$ со строками $U_{k}, k \in N_{s}$, принадлежит множеству $\Omega_{p}(\varphi(s, p))$. Согласно 
определению числа $\varphi(s, p)$ для любого разреза $x \notin P^{s}(W)$ найдется такой эффективный разрез $x^{0} \in P(x, W)$, что

$$
\left\|U_{k}\right\|_{p} \leqslant\|U\|_{p}<\varphi(s, p) \leqslant \frac{\left\langle W_{k}, \sigma\left(x^{0}, x\right)\right\rangle}{\left\|\sigma\left(x^{0}, x\right)\right\|_{q}}, \quad k \in N_{s} .
$$

Поэтому, применяя равенства (3) и неравенство Гельдера (1), для всякого индекса $k \in N_{s}$ выводим

$$
\begin{array}{r}
f_{k}\left(x^{0}, W_{k}+U_{k}\right)-f_{k}\left(x, W_{k}+U_{k}\right)=\left\langle W_{k}+U_{k}, \sigma\left(x^{0}, x\right)\right\rangle=\left\langle W_{k}, \sigma\left(x^{0}, x\right)\right\rangle+\left\langle U_{k}, \sigma\left(x^{0}, x\right)\right\rangle \geqslant \\
\geqslant\left\langle W_{k}, \sigma\left(x^{0}, x\right)\right\rangle-\left\|U_{k}\right\|_{p}\left\|\sigma\left(x^{0}, x\right)\right\|_{q}>0,
\end{array}
$$

т. е. разрез $x$ не принадлежит множеству Парето $P^{s}(W+U)$. Откуда заключаем, что при любой возмущающей матрице $U \in \Omega_{p}(\varphi(s, p))$ справедливо включение $P^{s}(W+$ $U) \subseteq P^{s}(W)$. Следовательно, верно неравенство $\rho(s, p) \geqslant \varphi(s, p)$.

Далее докажем неравенство $\rho(s, p) \leqslant \psi(s, p)$. Для этого сначала убедимся в справедливости неравенства $\rho(s, p) \leqslant(m s)^{1 / p} \varphi(s, \infty)$. В соответствии с определением величины $\varphi(s, \infty)$ найдется такой разрез $x^{0} \notin P^{s}(W)$, что для каждого эффективного разреза $x \in P\left(x^{0}, W\right)$ существует индекс $l \in N_{s}$, при котором

$$
\varphi(s, \infty)\left\|\sigma\left(x, x^{0}\right)\right\|_{1} \geqslant\left\langle W_{l}, \sigma\left(x, x^{0}\right)\right\rangle .
$$

Пусть $\varepsilon>(m s)^{1 / p} \varphi(s, \infty)$. Элементы $u_{k r}^{0}$ возмущающей матрицы $U^{0} \in \mathbf{R}^{s \times m}$ со строками $U_{k}^{0}=\left(u_{k 1}^{0}, u_{k 2}^{0}, \ldots, u_{k m}^{0}\right), k \in N_{s}$, зададим по правилу

$$
u_{k r}^{0}= \begin{cases}\delta, & \text { если } \gamma_{r}\left(x^{0}\right)=1, \\ -\delta, & \text { если } \gamma_{r}\left(x^{0}\right)=0,\end{cases}
$$

где $\varphi(s, \infty)<\delta<\varepsilon /(m s)^{1 / p}$. Тогда

$$
\begin{gathered}
\left\|U^{0}\right\|_{p}=\delta(m s)^{1 / p}, \quad U^{0} \in \Omega_{p}(\varepsilon), \\
\left\langle U_{k}^{0}, \sigma\left(x, x^{0}\right)\right\rangle=\sum_{r \in N_{m}} u_{k r}^{0}\left(\gamma_{r}(x)-\gamma_{r}\left(x^{0}\right)\right)=-\delta \sum_{r \in N_{m}}\left|\gamma_{r}(x)-\gamma_{r}\left(x^{0}\right)\right|= \\
=-\delta\left\|\sigma\left(x, x^{0}\right)\right\|_{1}, \quad k \in N_{s} .
\end{gathered}
$$

Из (4) и (5) с учетом (3) заключаем, что для каждого эффективного разреза $x \in$ $P\left(x^{0}, W\right)$ существует индекс $l \in N_{s}$, удовлетворяющий соотношениям

$$
\begin{array}{r}
f_{l}\left(x, W_{l}+U_{l}^{0}\right)-f_{l}\left(x^{0}, W_{l}+U_{l}^{0}\right)=\left\langle W_{l}+U_{l}^{0}, \sigma\left(x, x^{0}\right)\right\rangle=\left\langle W_{l}, \sigma\left(x, x^{0}\right)\right\rangle-\delta\left\|\sigma\left(x, x^{0}\right)\right\|_{1} \leqslant \\
\leqslant \varphi(s, \infty)\left\|\sigma\left(x, x^{0}\right)\right\|_{1}-\delta\left\|\sigma\left(x, x^{0}\right)\right\|_{1}<0 .
\end{array}
$$

Таким образом, справедлива формула

$$
\forall x \in P\left(x^{0}, W\right) \quad\left(x \notin X\left(x^{0}, W+U^{0}\right)\right) .
$$

Если $X\left(x^{0}, W+U^{0}\right)=\varnothing$, то $x^{0} \in P^{s}\left(W+U^{0}\right)$. Если же $X\left(x^{0}, W+U^{0}\right) \neq \varnothing$, то благодаря внешней устойчивости множества $P^{s}\left(W+U^{0}\right)$ (см., например, [18]) 
найдется такой разрез $x^{*} \in X\left(x^{0}, W+U^{0}\right)$, что $x^{*} \in P^{s}\left(W+U^{0}\right)$. Покажем, что $x^{*} \notin P^{s}(W)$.

Допустим обратное: $x^{*} \in P^{s}(W)$. Тогда согласно (6) выполняется включение $x^{*} \in P^{s}(W) \backslash P\left(x^{0}, W\right)$. Поэтому найдется индекс $l \in N_{s}$, при котором справедливо неравенство $f_{l}\left(x^{*}, W_{l}\right)<f_{l}\left(x^{0}, W_{l}\right)$. Отсюда, учитывая $(3)$ и $(5)$, приходим к соотношениям

$$
\begin{aligned}
& f_{l}\left(x^{*}, W_{l}+U_{l}^{0}\right)-f_{l}\left(x^{0}, W_{l}+U_{l}^{0}\right)=\left\langle W_{l}+U_{l}^{0}, \sigma\left(x^{*}, x^{0}\right)\right\rangle= \\
& \quad=\left\langle W_{l}, \sigma\left(x^{*}, x^{0}\right)\right\rangle-\delta\left\|\sigma\left(x^{*}, x^{0}\right)\right\|_{1}=f_{l}\left(x^{*}, W_{l}\right)-f_{l}\left(x^{0}, W_{l}\right)-\delta\left\|\sigma\left(x^{*}, x^{0}\right)\right\|_{1}<0,
\end{aligned}
$$

которые противоречат включению $x^{*} \in P^{s}\left(W+U^{0}\right)$.

Итак, при любом числе $\varepsilon>(m s)^{1 / p} \varphi(s, \infty)$ гарантируется существование такой возмущающей матрицы $U^{0} \in \Omega_{p}(\varepsilon)$, что $P^{s}\left(W+U^{0}\right) \nsubseteq P^{s}(W)$. Следовательно, $\rho(s, p)<\varepsilon$ для всякого $\varepsilon>(m s)^{1 / p} \varphi(s, \infty)$, тем самым $\rho(s, p) \leqslant(m s)^{1 / p} \varphi(s, \infty)$.

Наконец, убедимся в справедливости неравенства $\rho(s, p) \leqslant \chi(s, p)$. Пусть разрез $x^{0} \notin P^{s}(W)$ (тогда и $\overline{x^{0}} \notin P^{s}(W)$ ), а индекс $l \in N_{s}$ таков, что $\chi(s, p)=\left\|W_{l}\right\|_{p}$, где $W_{l}=\left(w_{l 1}, w_{l 2}, \ldots, w_{l m}\right)$. Задав число $\varepsilon>\chi(s, p)$, зафиксируем $\delta$, удовлетворяющее неравенствам

$$
0<\delta m^{1 / p}<\varepsilon-\chi(s, p)
$$

Введем в рассмотрение вспомогательный вектор $\xi=\left(\xi_{1}, \xi_{2}, \ldots, \xi_{m}\right)$, каждая компонента которого $\xi_{r}, r \in N_{m}$, имеет вид

$$
\xi_{r}= \begin{cases}\delta, & \text { если } \gamma_{r}\left(x^{0}\right)=1, \\ -\delta, & \text { если } \gamma_{r}\left(x^{0}\right)=0 .\end{cases}
$$

Тогда очевидно равенство (ср. с (5))

$$
\left\langle\xi, \sigma\left(x, x^{0}\right)\right\rangle=-\delta\left\|\sigma\left(x, x^{0}\right)\right\|_{1} .
$$

Теперь строки $U_{k}^{0} \in \mathbf{R}^{m}, k \in N_{s}$, возмущающей матрицы $U^{0} \in \mathbf{R}^{s \times m}$ зададим по правилу

$$
U_{k}^{0}= \begin{cases}\xi-W_{l}, & \text { если } k=l, \\ \mathbf{0}^{(m)}, & \text { если } k \neq l .\end{cases}
$$

В результате, учитывая (8), для всякого разреза $x \in X \backslash\left\{x^{0}, \overline{x^{0}}\right\}$ имеем

$$
\left\langle U_{l}^{0}, \sigma\left(x, x^{0}\right)\right\rangle=-\delta\left\|\sigma\left(x, x^{0}\right)\right\|_{1}-\left\langle W_{l}, \sigma\left(x, x^{0}\right)\right\rangle,
$$

и благодаря (7) находим

$$
\left\|U^{0}\right\|_{p}=\left\|U_{l}^{0}\right\|_{p}=\left\|\xi-W_{l}\right\|_{p} \leqslant\|\xi\|_{p}+\left\|W_{l}\right\|_{p}=\delta m^{1 / p}+\left\|W_{l}\right\|_{p}=\delta m^{1 / p}+\chi(s, p)<\varepsilon .
$$

Отсюда для каждого разреза $x \in X \backslash\left\{x^{0}, \overline{x^{0}}\right\}$ получаем

$$
f_{l}\left(x, W_{l}+U_{l}^{0}\right)-f_{l}\left(x^{0}, W_{l}+U_{l}^{0}\right)=\left\langle W_{l}+U_{l}^{0}, \sigma\left(x, x^{0}\right)\right\rangle=-\delta\left\|\sigma\left(x, x^{0}\right)\right\|_{1}<0,
$$

т. е. $x \notin X\left(x^{0}, W+U^{0}\right)$. Так как разрезы $x^{0}$ и $\overline{x^{0}}$ не содержатся в $X\left(x^{0}, W+U^{0}\right)$, то $X\left(x^{0}, W+U^{0}\right)=\varnothing$. Это значит, что $x^{0} \in P^{s}\left(W+U^{0}\right)$, где $U^{0} \in \Omega_{p}(\varepsilon)$. Так как $x^{0} \notin P^{s}(W)$, то заключаем, что $\rho(s, p) \leqslant \varepsilon$ для любого числа $\varepsilon>\chi(s, p)$. 
Следовательно, $\rho(s, p) \leqslant \chi(s, p)$, что вместе с доказанным выше неравенством $\rho(s, p) \leqslant(m s)^{1 / p} \varphi(s, \infty)$ дает требуемую верхнюю оценку $\rho(s, p) \leqslant \psi(s, p)$.

Теорема 1 доказана.

Из теоремы 1 вытекает следующее утверждение о достижимости нижней и верхней оценок радиуса устойчивости задачи $Z^{s}(W)$ при $p=\infty$.

Следствие 1. Для радиуса устойчивости нетривиальной задачи $Z^{s}(W), s \in \mathbf{N}$, справедливы следующие равенства

$$
\rho(s, \infty)=\varphi(s, \infty)=\psi(s, \infty)=\min _{x \in X \backslash P^{s}(W)} \max _{x^{\prime} \in P(x, W)} \min _{k \in N_{s}} \frac{\left\langle W_{k}, \sigma\left(x^{\prime}, x\right)\right\rangle}{\left\|\sigma\left(x^{\prime}, x\right)\right\|_{1}} .
$$

О достижимости нижней оценки радиуса устойчивости $\varphi(s, p)$ свидетельствует следующее утверждение.

Следствие 2. При любых $s \in \mathbf{N} u p \in[1, \infty]$ существует такой класс нетривиальных задач $Z^{s}(W)$, что

$$
\rho(s, p)=\varphi(s, p) .
$$

Доказательство. Будем рассматривать класс задач с условием $P^{s}(W)=$ $\left\{x^{0}, \overline{x^{0}}\right\}$. Тогда

$$
\varphi=\varphi(s, p)=\min _{x \in X \backslash\left\{x^{0}, \overline{x^{0}}\right\}} \min _{k \in N_{s}} \frac{\left\langle W_{k}, \sigma\left(x^{0}, x\right)\right\rangle}{\left\|\sigma\left(x^{0}, x\right)\right\|_{q}} .
$$

В силу теоремы 1 для доказательства равенства $\rho(s, p)=\varphi$ достаточно показать, что $\rho(s, p) \leqslant \varphi$. Этому посвящено дальнейшее изложение .

Согласно определению величины $\varphi$ найдутся такие $x^{*} \notin P^{s}(W)$ и $l \in N_{s}$, что

$$
\varphi\left\|\sigma\left(x^{0}, x^{*}\right)\right\|_{q}=\left\langle W_{l}, \sigma\left(x^{0}, x^{*}\right)\right\rangle .
$$

Зададим $\varepsilon>\varphi$ и зафиксируем число $\theta$, подчиненное неравенствам $\varphi<\theta<\varepsilon$. Согласно формуле (2) существует такой вектор $u^{0} \in \mathbf{R}^{m}$, что

$$
\left\|u^{0}\right\|_{p}=\theta \quad \& \quad\left\langle u^{0}, \sigma\left(x^{0}, x^{*}\right)\right\rangle=-\theta\left\|\sigma\left(x^{0}, x^{*}\right)\right\|_{q} .
$$

Поэтому, задав строки $U_{k}^{0}, k \in N_{s}$, возмущающей матрицы $U^{0} \in \mathbf{R}^{s \times m}$ по правилу

$$
U_{k}^{0}= \begin{cases}u^{0}, & \text { если } k=l, \\ \mathbf{0}^{(m)}, & \text { если } k \neq l,\end{cases}
$$

имеем

$$
\left\|U^{0}\right\|_{p}=\theta, \quad\left\langle U_{l}^{0}, \sigma\left(x^{0}, x^{*}\right)\right\rangle=-\theta\left\|\sigma\left(x^{0}, x^{*}\right)\right\|_{q} .
$$

Отсюда и из (9) с учетом (3) получаем

$f_{l}\left(x^{0}, W_{l}+U_{l}^{0}\right)-f_{l}\left(x^{*}, W_{l}+U_{l}^{0}\right)=\left\langle W_{l}+U_{l}^{0}, \sigma\left(x^{0}, x^{*}\right)\right\rangle=\left\langle W_{l}, \sigma\left(x^{0}, x^{*}\right)\right\rangle-\theta\left\|\sigma\left(x^{0}, x^{*}\right)\right\|_{q}<0$.

Таким образом, оба разреза $x^{0}$ и $\overline{x^{0}}$ не содержатся в $X\left(x^{*}, W+U^{0}\right)$. Далее, вновь принимая во внимание внешнюю устойчивость множества Парето и рассуждая аналогично доказательству теоремы 1 , приходим к выводу, что $P^{s}\left(W+U^{0}\right) \nsubseteq P^{s}(W)$, где $\varphi<\left\|U^{0}\right\|_{p}<\varepsilon$. Следовательно, $\rho(s, p) \leqslant \varphi$.

Следствие 2 доказано. 
Следствие 3. При любом $p \in[1, \infty]$ для радиуса устойчивости нетривиальной скалярной задачи $Z^{1}(W), W \in \mathbf{R}^{1 \times m}$, справедливы оценки

$$
\varphi(1, p) \leqslant \rho(1, p) \leqslant \min \left\{\|W\|_{p}, m^{1 / p} \varphi(1, \infty)\right\} .
$$

Следствие 4. При любых $s \in \mathbf{N} u p \in[1, \infty]$ радиус устойчивости $\rho(s, p)$ нетривиальной задачи $Z^{s}(W)$ положителен (задача $Z^{s}(W)$ устойчива) тогда и только тогда, когда $\varphi(s, p)>0$.

\section{Список литературы}

1. Емеличев В. А., Кричко В. Н., Подкопаев Д. П., О радиусе устойчивости векторной задачи линейного булева программирования. Дискретная математика (2000) 12, №2, $25-30$.

2. Emelichev V. A., Girlich E., Nikulin Yu. V., Podkopaev D. P., Stability and regularization of vector problems of integer linear programming. Optimization (2002) 51, №4, 645-676.

3. Бухтояров С. Е., Емеличев В. А., Мера устойчивости конечной коалиционной игры с параметрическим («от Парето от Нэша») принципом оптимальности ЖКурнал вычисл. матем. и матем. физики (2006) 46, №7, 1274-1280.

4. Емеличев В. А., Кузьмин К. Г., Об одном типе устойчивости многокритериальной задачи целочисленного линейного программирования в случае монотонной нормы. Известия РАН. Теория и системы управления (2007) №5, 45-51.

5. Емеличев В. А., Кузьмин К. Г., О радиусе устойчивости векторной задачи целочисленного линейного программирования в случае в случае регулярности нормы в критериальном пространстве. Кибернетика и системный анализ (2010) №1, 82-89.

6. Emelichev V., Podkopaev D., Quantitative stability analysis for vector problems of 0-1 programming. Discrete Optimization (2010) 7, 48-63.

7. Емеличев В. А., Кричко В. Н., Формула радиуса устойчивости векторной $l_{\infty}$ экстремальной траекторной задачи. Дискретная математика (2004) 16, №1, 14-20.

8. Emelichev V., Korotkov V., On stability radius of the multicriteria variant of Markowitz's investment portfolio problem. Buletinul Academiei de ştiinte a Respublicii Moldova. Matematica (2011) №1, 83-94.

9. Емеличев В. А., Коротков В. В., О радиусе устойчивости векторной инвестиционной задачи с критериями минимаксного риска Сэвиджа. Кибернетика и системный анализ (2012) №3, 68-77.

10. Емеличев В. А., Коротков В. В., Устойчивость векторной инвестиционной булевой задачи с критериями Вальда. Дискретная математика (2012) 24, №3. 3-16.

11. Гэри М., Джонсон Д., Вычислительные машины и труднорешаемые задачи. Мир, Москва, 1982.

12. Burer S., Monteiro R. D. C., Zhang Y., Rank-two relaxation heuristics for MAX-CUT and other binary quadratic programs. SIAM Journal on Optimization (2001) 12, 503-521.

13. Barahona F., Grotschel M., Junger M., Reinelt G., An application of combinatorial optimization to statistical physics and circuit layout design. Operations Research (1988) 36, №3, 493-513.

14. Chang K. C., Du D. H.-C., Efficient algorithms for layer assignment problem. IEEE Transactions on Computer-Aided Design of Integrated Circuits and Systems (1987) 6, 67-78.

15. Poljac S., Tuza Z., Maximum cuts and large bipartite subgraphs. DIMACS Series in Discrete Mathematics and Theoretical Computer Science (1995) 20, 181-244. 
16. Емеличев В. А., Мельников О. И., Сарванов В. И., Тышкевич Р. И., Лекции по теории графов. ЛИБРОКОМ, Москва, 2009.

17. Харди Г., Литтльвуд Дж. Е., Полиа Г., Неравенства. ЛКИ, Москва, 2008.

18. Подиновский В. В., Ногин В. Д., Парето-оптималънъе решения многокритериалънъх задач. ФИЗМАТЛИТ, Москва, 2007.

Статья поступила 15.01.2013. 